\title{
ESTRATEGIAS COGNITIVAS Y METACOGNITIVAS EN ESTUDIANTES DE EDUCACIÓN SECUNDARIA CON APTITUDES SOBRESALIENTES
}

\section{COGNITIVE AND METACOGNITIVE STRATEGIES IN HIGH SCHOOL STUDENTS WITH OUTSTANDING SKILLS}

\author{
Norma Guadalupe Márquez Cabellos \\ Adriana Isabel Andrade Sánchez \\ Julio Cuevas Romo
}

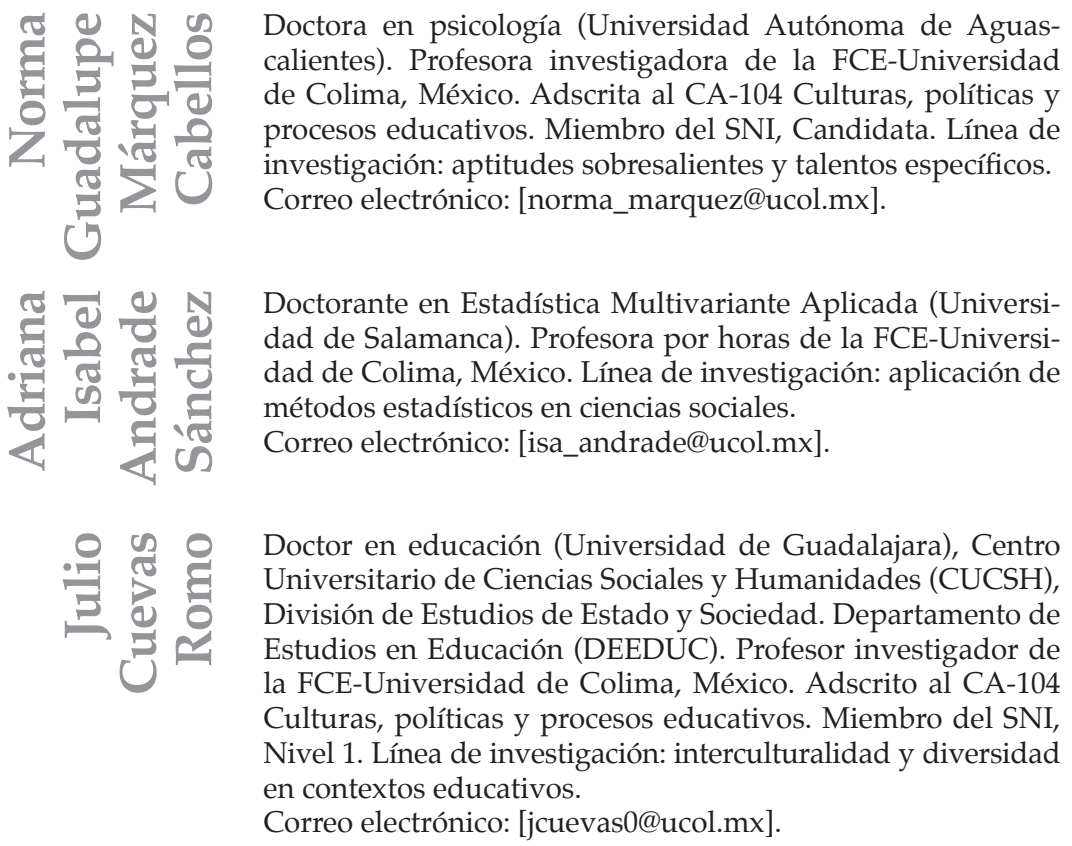




\title{
RESUMEN
}

El presente artículo aborda un estudio realizado a 254 adolescentes de educación secundaria con aptitudes sobresalientes, centrado en la identificación de estrategias cognitivas y estrategias metacognitivas bajo la configuración del Cuestionario de Estrategias de Aprendizaje -CEA- (Beltrán, Pérez y Ortega, 2006). La investigación es de corte cuantitativo. Los resultados identificaron el perfil de aprendizaje, las estrategias cognitivas y metacognitivas implementadas antes, durante y después de la solución a un problema; lo que permitió afirmar la importancia de desarrollar estrategias cognitivas y metacognitivas, encaminadas a adquirir herramientas para el trabajo autónomo, el aprendizaje autorregulado, así como la planeación, evaluación y el control del propio aprendizaje; conduciendo a obtener éxito en diferentes áreas de estudio.

Palabras clave: estrategias cognitivas, estrategias metacognitivas, aptitudes sobresalientes.

\begin{abstract}
This article addresses a study of 254 high school adolescents with outstanding skills, focusing on the identification of cognitive strategies and metacognitive strategies from the perspective of the Learning Strategies Questionnaire -CEA- (Beltrán, Pérez and Ortega, 2006). The research is quantitative. The results allow to identify the learning profile, the applied cognitive and metacognitive strategies previously, during and after resolve a problem; which made it possible to affirm the importance of developing cognitive and metacognitive strategies aimed at acquiring tools for autonomous work, self-regulated work, planning, evaluation and self-learning control, to get success in different study areas.
\end{abstract}

Key words: cognitive strategies, metacognitive strategies, outstanding skills. 


\section{INTRODUCCIÓN}

La participación de México en la Conferencia mundial sobre educación para todos «Satisfacción de las necesidades básicas de aprendizaje» realizada en Jomtiem, Tailandia en 1990, en la Declaración de principios, políticas y prácticas para las necesidades educativas especiales efectuada en Salamanca, España en 1994, y en la Conferencia nacional "Atención educativa a menores con necesidades educativas especiales: Equidad para la diversidad» celebrada en Huatulco, Oaxaca en 1997, ha permitido impulsar una educación que responda a la diversidad de los alumnos en las aulas regulares, potencializando y promoviendo al máximo las capacidades y habilidades de alumnos de educación básica.

Sin embargo, la variedad de prácticas pedagógicas, las imprecisiones en la implementación de la reforma educativa, la falta de recursos y/o apoyos específicos, así como la identificación de las necesidades y la condición de los alumnos, han sido barreras para que sean una realidad en las escuelas; falta aún un largo camino por recorrer.

Como respuesta al principio de equidad educativa, el Congreso General de los Estados Unidos Mexicanos promulga en mayo de 2011 la Ley general para la inclusión de las personas con discapacidad; donde se especifica que el Estado debe proteger y asegurar los derechos de las personas con discapacidad, asegurando su plena inclusión en la sociedad en un marco de respeto, igualdad y equiparación de oportunidades. Bajo este decreto, los niveles de educación básica enfrentan el reto de satisfacer las necesidades educativas especiales y específicas de sus alumnos, independientemente de las capacidades, habilidades, destrezas y/o dificultades que manifiesten, es decir; es esencial considerar a la población que presenta una discapacidad y/o trastorno, así como aquellos que destacan por manifestar aptitudes sobresalientes y talentos específicos.

Atender a la diversidad concierne a todos los niños y adolescentes; por ello, este artículo centra su atención en la identificación de las estrategias cognitivas y estrategias metacognitivas de estudiantes de educación secundaria con aptitudes sobresalientes. Referimos la importancia de su identificación e intervención educativa para 
garantizar el desarrollo de habilidades que los conduzcan a un aprendizaje autónomo, reflexivo, analítico y autorregulado.

Dentro del mundo de las altas habilidades, los alumnos han sido comprendidos de diferentes formas a lo largo de la historia, desde visiones más simples a propuestas complejas en las que se refleja el fenómeno como sistema de características que interactúan entre sí. Para efectos de esta investigación, nos referiremos como «aptitudes sobresalientes» - porque es un término conocido por el profesorado y construido en nuestro país en la Propuesta de intervención educativa: atención educativa a alumnos y alumnas con aptitudes sobresalientes (SEP, 2006)-, refiriéndose a los alumnos como:

[...] aquéllos capaces de destacar significativamente del grupo social y educativo al que pertenecen en uno o más de los siguientes campos del quehacer humano: científico-tecnológico, humanístico social, artístico o acción motriz. Estos alumnos, por presentar necesidades específicas, requieren de un contexto facilitador que les permita desarrollar sus capacidades personales y satisfacer sus necesidades e intereses para su propio beneficio y el de la sociedad (SEP, 2006, p. 59).

Existe la creencia que esta población sobrevive y triunfa automáticamente, que sus capacidades y habilidades se manifiestan por sí mismas; que no requieren de apoyos específicos ante cualquier dificultad o carencia que tengan. Esta confusión lleva generalmente a un desinterés por la identificación de sus necesidades educativas, habilidades, características y destrezas y, por ende, a la puesta en práctica de programas de intervención educativa que satisfagan sus intereses y motivaciones; quedan así postergados los compromisos pactados en una educación de igualdad de oportunidades para todos los alumnos.

Para efectos de este trabajo nos centraremos en un aspecto del aprendizaje de los estudiantes de educación secundaria con aptitudes sobresalientes: las estrategias cognitivas y estrategias metacognitivas. Para Flavell (1976), la cognición y metacognición se refiere al «conocimiento que uno tiene sobre los propios procesos y productos cognitivos o sobre cualquier cosa relacionada con ellos, es decir, las propiedades de la información o los datos relevantes para el aprendizaje» (p. 232). Beltrán 
(1993) refiere que los alumnos con altas habilidades utilizan estrategias cognitivas y metacognitivas, lo que explica el logro de un buen rendimiento académico. Sin embargo, prevalece la necesidad de ofrecer una educación que permita el desarrollo pleno de sus habilidades mentales. En sus estudios, Beltrán (1993) puntualiza que las estrategias metacognitivas «están relacionadas con el conocimiento que tiene cada persona de sus propios procesos de conocimiento; es el conocimiento del conocimiento» (p. 66). Es la puesta en práctica de la reflexión del alumno sobre la naturaleza de la tarea que debe emprender, es decir, observa y examina el procedimiento a ejecutar conduciéndolo a planear, regular y evaluar su aprendizaje. Por otra parte, este autor refiere que las estrategias cognitivas contribuyen considerablemente a realizar la tarea de la mejor manera posible, marcando el estilo intelectual por la forma habitual de aplicar las acciones de organización y selección de la información.

Con fundamento en sus estudios, Beltrán (2003) señala que las estrategias cognitivas y metacognitivas nos permiten reflexionar sobre la naturaleza de la tarea que tenemos que ejecutar, los objetivos planteados, las estrategias a utilizar para llevarla a cabo, así como las posibles dificultades que pudiéramos encontrar. Asimismo, señala que las investigaciones arrojan un cambio paradigmático y prometedor, alejado de los estudios tradicionales de capacidad estratégica en los niños y adolescentes que presentan altas habilidades. Refiere que se ha demostrado que los sujetos con aptitudes sobresalientes son cognitivamente superiores en el conocimiento, adquisición, flexibilidad y uso adecuado de estrategias; pero ocasionalmente se parecen a las de los sujetos que no tienen aptitudes sobresalientes al manifestar dificultades en el momento de utilizar nuevas estrategias a las ya aprendidas.

Beltrán y Genovard (1996) concluyen que existen estudiantes que carecen de algunas habilidades o estrategias básicas para el aprendizaje, siendo necesario capacitarles para ellas, orientándolos a aprender las reglas para aprender. De lo contrario, no serán capaces de alcanzar su potencial si no han sido instruidos. Las estrategias de aprendizaje son las herramientas idóneas para construir el conocimiento, cuantas más y mejor estrategias utilice un alumno en su aprendizaje, mayor será su nivel de rendimiento (Beltrán y Genovard, 1996). 
A pesar de que son múltiples los estudios internacionales centrados en la aplicación de las estrategias metacognitivas en estudiantes de Educación Básica, Media Superior y Educación Superior, el de mayor antigüedad —identificado hasta el momento - es el de Ugartetxea (1996), quien realizó un estudio acerca de la capacidad transferencial de la metacognición y su influencia en el rendimiento intelectual con la aplicación del programa Orientación Metacognitiva de la Comprensión Lectora (OMECOL), con alumnos de Enseñanza General Básica del sistema español. El objetivo fue considerar si realmente surge una transferencia de lo adquirido mediante orientación metacognitiva a otras aptitudes escolares, además de la propia comprensión, a la par de tratar de comprobar si existe una asociación entre metacognición e inteligencia. Los resultados confirmaron la existencia de una asociación entre metacognición y rendimiento intelectual. Los datos obtenidos también permiten aceptar la consideración de que la orientación metacognitiva de la comprensión lectora mejora el rendimiento en actividades académicas diferentes a aquéllas para la cuales fueron inicialmente diseñadas.

En el estudio realizado por Pomar, Díaz y Fernández (2006), cuyo objetivo fue la aplicación de un Programa de Enriquecimiento con alumnos superdotados, se demostró que esta población presenta mayor capacidad y rapidez para resolver problemas: analizando, sintetizando y dedicando más tiempo a la planificación que a la resolución misma. En sus investigaciones Davidson y Sternberg (1984) señalan que los alumnos con alto rendimiento académico tienen mejor conocimiento de estrategias resolutivas que otros alumnos. Por su parte, Shore (2000) refiere en sus estudios que son más consistentes, adaptativos y eficaces para escoger sus estrategias cognitivas y metacognitivas.

En España, Valle, Núñez, Cabanach, Rodríguez, González y Rosario (2009) llevaron a cabo un estudio evaluando las metas cognitivas con el Cuestionario para la Evaluación de Metas Académicas en Secundaria (CEMA-II). Las metas académicas se valoraron con el Cuestionario de Estrategias Cognitivas de Aprendizaje y Estudio (CECAE) que evaluaban las estrategias cognitivas (selección, organización, elaboración y memorización de información). El objetivo de dicha investigación estuvo centrado en conocer las posibles diferencias en el 
uso de estrategias cognitivas y de autorregulación ${ }^{1}$ del proceso de estudio, entre estudiantes de educación secundaria con diferentes tipos de motivaciones académicas. En los resultados con relación a las metas de aprendizaje centradas en la adquisición de competencias y control, se aprecian diferencias significativas en el uso de cada una de las estrategias, tanto cognitivas como de autorregulación del estudio. Cuanto más altas son este tipo de metas, mayor es el uso que hacen los estudiantes de las diferentes estrategias cognitivas y de autorregulación del estudio, y cuanto más bajas son las metas, menor es el empleo que hacen de esas estrategias.

Por su parte, Barca-Lozano, Almeida, Porto-Rioboo, PeralboUzquiano, y Brenlla-Blanco (2012), llevaron a cabo una investigación cuyo objetivo fue analizar el impacto de variables personales relacionadas con las metas académicas y las estrategias de aprendizaje del alumnado de educación secundaria en su rendimiento académico. La relevancia de la investigación reside en la importancia que las variables motivacionales poseen sobre el rendimiento en contextos escolares. Los resultados de dicha investigación sugieren que las metas académicas de aprendizaje y las estrategias de autoeficacia pueden asumirse como factores determinantes positivos del rendimiento académico, existiendo también un impacto, pero en sentido negativo, de las metas de valoración social y las estrategias superficiales de aprendizaje.

Por otra parte, Sánchez y Pulgar en el año 2014, realizaron un estudio con estudiantes universitarios para identificar el impacto de un programa de intervención, centrado en estrategias de aprendizaje (cognitivas y metacognitivas), intereses, realizaciones creativas y en el rendimiento académico. Los resultados indicaron que obtienen mejores resultados en el rendimiento académico quienes emplean las estrategias metacognitivas porque desarrollan la estrategia de procesamiento profundo que repercute en el rendimiento académico.

1 «La autorregulación del aprendizaje hace referencia a los procesos individuales mediante los cuales los alumnos activan, orientan y mantienen sus pensamientos, afectos y acciones hacia la consecución de objetivos de aprendizaje» (Monereo y Badia, 2013, p. 23). Posibilita a las personas funcionar de manera eficaz, adquiriendo los conocimientos y las destrezas que necesitan en el contexto escolar, áulico y en su efecto en el mundo laboral. 
Pifarré y Sanuy (2001) mencionan que estudios científicos han demostrado que los buenos resolutores de problemas son aquéllos caracterizados por poseer un conjunto de estrategias generales, superando las dificultades que encuentran durante ese proceso. Por tanto, el uso de las estrategias apropiadas favorece la adquisición de aprendizajes de mayor calidad, dotando a los alumnos de herramientas para el aprendizaje.

En sus estudios, Beltrán (2003) puntualiza que los estudiantes con alto rendimiento académico disponen de estrategias superiores para el conocimiento, la adquisición, flexibilidad y el uso adecuado de estrategias, pero sus habilidades estratégicas a veces parecen similares a las de sus compañeros regulares. También señala que la identificación de las estrategias de aprendizajes empleadas por los alumnos, permitirá diagnosticar las causas de la diferencia entre un rendimiento escolar y el mejoramiento de su aprendizaje.

\section{MÉTODO}

A pesar de que son múltiples los estudios internacionales centrados en los referentes cognitivos y metacognitivos de los alumnos con altas capacidades intelectuales, sigue repercutiendo la necesidad de continuar con exploraciones con el fin de comprender sus procesos y funcionamiento. Los beneficios que pueden obtenerse son variados porque antes de enseñar una estrategia de aprendizaje a un alumno, o bien, antes de diseñar un programa de entrenamiento cognitivo y metacognitivo, es importante identificar el conocimiento que tiene el alumno de las estrategias y la eficacia de las mismas en su rendimiento académico al momento de planear, autorregular y evaluar su aprendizaje. Por ello, el objetivo de la investigación se centró en identificar las estrategias cognitivas y metacognitivas de adolescentes de educación secundaria con aptitudes sobresalientes.

La investigación fue de corte cuantitativo por la recolección de datos obtenidos en el instrumento de medición aplicado. La muestra se conformó por 254 adolescentes con aptitudes sobresalientes (131 mujeres y 
123 hombres) pertenecientes a la Escuela Secundaria Estatal número 12, del Estado de Colima, en México. Esta institución es conocida como «Escuela de talentos» por su modalidad de semi-internado; y se centra en la intervención educativa de agrupación para estudiantes con aptitudes sobresalientes y talentos específicos. Es importante referir que es única en nuestro país.

Para la identificación de las estrategias cognitivas y metacognitivas se aplicó el Cuestionario de Estrategias de Aprendizaje - CEA $^{2}$ - (Beltrán, Pérez y Ortega, 2006), el cual valora el nivel de utilización de las estrategias de aprendizaje. Se compone de 70 reactivos que evalúan 4 escalas y 11 subescalas en las que se agrupan las siguientes estrategias cognitivas y metacognitivas: (a) sensibilización: motivación, actitud, control emocional; (b) elaboración: selección de la información, organización, elaboración de información; (c) personalización: pensamiento crítico y creativo, recuperación, transferencia, y (d) metacognición: planificación, evaluación y regulación.

Las orientaciones otorgadas a los estudiantes, según las puntuaciones obtenidas en el CEA, pueden agruparse en tres partes, de acuerdo con los autores. Esta categorización depende de los percentiles de la población de estudio:

Tabla 1. Puntos de corte para determinar las orientaciones para el alumno.

\section{Estrategias}

\section{Con nivel adecuado y posibilidades de mejora}

Desarrolladas adecuadamente
Percentil $<25$
$\geq 25 y \leq 60$ $>60$

2 La finalidad del «Cuestionario de estrategias de aprendizaje» es evaluar el nivel de utilización de las estrategias de aprendizaje por los estudiantes y ofrecer orientaciones de distinto nivel - tanto para profesores como para alumnos - de forma que los implicados en el proceso educativo inicien un programa para potenciar fuerzas y compensar debilidades (Beltrán, Pérez y Ortega, 2006). 
Los datos fueron analizados empleando el Statistical Package for the Social Sciences (SPSS v.20) para obtener los puntos de corte, las tablas de frecuencia, así como la asociación de Spearman. Para analizar en conjunto las subescalas que conforman el CEA, se realizó un HJ-Biplot, técnica desarrollada por Galindo y Vicente-Villardón (1986), utilizando MultBiplot (Vicente-Villardón v 15.1214, 2014). El Biplot, es una representación gráfica de una matriz de datos X(nxp), resultante de observar $n$ individuos en $p$ características numéricas (Gabriel, 1971). El HJ-Biplot es una representación gráfica multivariante; los marcadores se han elegido de manera que ambos pueden ser superpuestos en un mismo sistema de referencia, con máxima calidad de representación, mostrando de modo conjunto individuos y variables de una matriz cualquiera (Galindo y Vicente-Villardón, 1986).

\section{RESULTADOS}

Beltrán, Pérez y Ortega (2006) establecen que los adolescentes que hayan obtenido puntuaciones inferiores al percentil 25, necesitan recibir ayuda de sus tutores para mejorar su aprendizaje; aquellos estudiantes con puntuaciones entre los centiles 25 y 60 , se encuentran en un nivel adecuado y con posibilidades de mejora; los estudiantes con puntuaciones superiores a los centiles 60, deben ser animados a continuar con su práctica, ya que no precisan intervención específica, porque las estrategias están siendo desarrolladas adecuadamente. En los resultados obtenidos por los estudiantes con aptitudes sobresalientes se procedió a calcular los puntos de corte para determinar el nivel en que se encuentran y, de esta forma, descubrir áreas de oportunidad así como líneas de acción: 
Tabla 2. Puntos de corte para las subescalas del CEA en estudiantes con aptitudes sobresalientes.

\begin{tabular}{|c|c|c|c|c|}
\hline Escala & Subescala & $\begin{array}{c}\text { Necesitan } \\
\text { mejorar }\end{array}$ & $\begin{array}{c}\text { Con nivel adecuado } \\
\text { y posibilidades de } \\
\text { mejora }\end{array}$ & $\begin{array}{l}\text { Desarrolladas } \\
\text { adecuadamente }\end{array}$ \\
\hline \multirow{3}{*}{$\begin{array}{l}\text { Sensibili- } \\
\text { zación }\end{array}$} & Motivación & $<45$ & $\geq 45 y \leq 70$ & $>70$ \\
\hline & Actitud & $<15$ & $\geq 15 y \leq 60$ & $>60$ \\
\hline & $\begin{array}{c}\text { Control } \\
\text { emocional }\end{array}$ & $<30$ & $\geq 30 y \leq 75$ & $>75$ \\
\hline \multirow{3}{*}{$\begin{array}{l}\text { Elabo- } \\
\text { ración }\end{array}$} & Elaboración & $<40$ & $\geq 40 y \leq 80$ & $>80$ \\
\hline & Organización & $<13$ & $\geq 13 y \leq 45$ & $>45$ \\
\hline & Selección & $<25$ & $\geq 25 \mathrm{y} \leq 70$ & $>70$ \\
\hline \multirow{3}{*}{$\begin{array}{l}\text { Personali- } \\
\text { zación }\end{array}$} & Transferencia & $<35$ & $\geq 35 y \leq 80$ & $>80$ \\
\hline & $\begin{array}{l}\text { Pensamiento } \\
\text { crítico }\end{array}$ & $<45$ & $\geq 45 y \leq 85$ & $>85$ \\
\hline & Recuperación & $<15$ & $\geq 15 y \leq 45$ & $>45$ \\
\hline \multirow{2}{*}{$\begin{array}{l}\text { Metacog- } \\
\text { nición }\end{array}$} & Planificación & $<26.75$ & $\geq 26.75 y \leq 65$ & $>65$ \\
\hline & Regulación & $<15$ & $\geq 15 y \leq 40$ & $>40$ \\
\hline
\end{tabular}

Una vez calculados los puntos de corte para nuestra población, se analizan los resultados obtenidos (ver tabla 3). Como es posible observar, las subescalas que más estudiantes necesitan mejorar son: planificación (25\%), organización (24\%) y pensamiento crítico $(24 \%)$; las escalas mejor desarrolladas son: control emocional, planificación y regulación (38\%). La mayoría de los estudiantes poseen un nivel adecuado, aunque con posibilidades de mejora; las subescalas con mayores puntajes en esta situación son: actitud (49\%), selección $(46 \%)$ y recuperación $(46 \%)$. 
Tabla 3. Distribución de los estudiantes según las orientaciones necesarias y las subescalas del CEA.

\begin{tabular}{lcccccc}
\hline & \multicolumn{3}{c}{ Necesita mejorar } & $\begin{array}{c}\text { Nivel adecuado y } \\
\text { con posibilidades } \\
\text { de mejora }\end{array}$ & $\begin{array}{c}\text { Desarrollada } \\
\text { adecuadamente }\end{array}$ \\
\cline { 2 - 7 } Subescalas & $\mathrm{n}$ & $\%$ & $\mathrm{n}$ & $\%$ & $\mathrm{n}$ & $\%$ \\
Motivación & 54 & $21 \%$ & 110 & $43 \%$ & 89 & $35 \%$ \\
\hline Actitud & 48 & $19 \%$ & 124 & $49 \%$ & 82 & $32 \%$ \\
\hline Control emocional & 55 & $22 \%$ & 102 & $40 \%$ & 97 & $38 \%$ \\
\hline Elaboración & 59 & $23 \%$ & 114 & $45 \%$ & 81 & $32 \%$ \\
\hline Organización & 62 & $24 \%$ & 105 & $41 \%$ & 87 & $34 \%$ \\
\hline Selección & 55 & $22 \%$ & 117 & $46 \%$ & 82 & $32 \%$ \\
\hline Transferencia & 52 & $20 \%$ & 115 & $45 \%$ & 87 & $34 \%$ \\
\hline Pensamiento crítico & 62 & $24 \%$ & 112 & $44 \%$ & 80 & $31 \%$ \\
\hline Recuperación & 44 & $17 \%$ & 117 & $46 \%$ & 92 & $36 \%$ \\
\hline Planificación & 63 & $25 \%$ & 94 & $37 \%$ & 97 & $38 \%$ \\
\hline Regulación & 52 & $20 \%$ & 106 & $42 \%$ & 96 & $38 \%$ \\
\hline
\end{tabular}

Aunque las escalas corresponden a los cuatro grandes procesos del aprendizaje humano complejo (Beltrán, 1993, citado por Beltrán, Pérez y Ortega, 2006):

[...] en realidad se trata de una verdadera cadena procesual cognitiva en la que los diversos momentos procesuales están íntimamente relacionados entre sí y sólo se pueden separar a efectos de elaboración mental y de aplicación instruccional (Beltrán, 2003, p. 9). 
Esta afirmación puede demostrarse por medio del HJ-Biplot mostrado en la figura 1, donde se comprueba la relación existente entre las puntuaciones que los estudiantes proporcionaron a las subescalas del CEA.

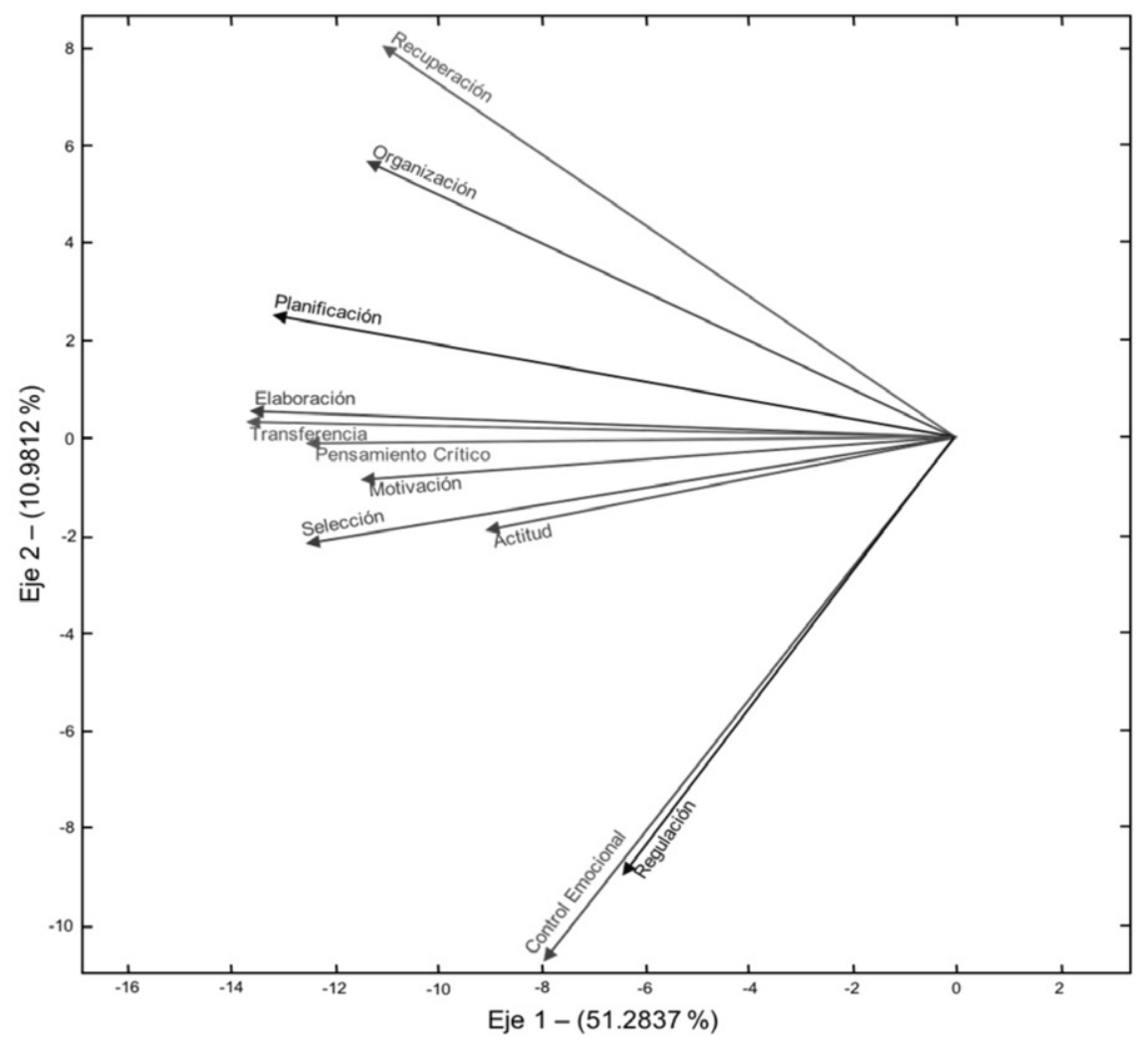

Figura 1. HJ-Biplot de los puntajes obtenidos por los estudiantes con aptitudes sobresalientes en las escalas del CEA.

En el HJ-BIplot se observa que los alumnos que puntuaron alto en los ítems conformados por la subescala control emocional, también lo hicieron en los ítems de la subescala de regulación y viceversa, mostrando así la interrelación entre metacognición y sensibilización. De 
igual forma, puede verse cómo la subescala de selección (perteneciente a la escala de elaboración) se relaciona fuertemente con la actitud y la motivación (de la escala de sensibilización); es decir, existe la posibilidad de que al trabajar con temas de selección, la actitud y motivación de los estudiantes mejoren significativamente (ver figura 1).

Asimismo, en el HJ-Biplot se observa la relación positiva intensa entre los puntajes otorgados a los ítems que conforman las subescalas elaboración, transferencia, pensamiento crítico, motivación y selección. Por tratarse de subescalas altamente relacionadas, es posible que trabajar con alguna de estas subescalas permita obtener un impacto positivo en las demás.

En el análisis del HJ-Biplot, cabe destacar cómo la subescala planificación posee una fuerte relación positiva con la subescala elaboración, y moderada con la de organización. De igual forma se rescata que la subescala recuperación mantiene una fuerte relación positiva con la subescala organización, por lo que se espera que, al trabajar con los estudiantes en temas de organización, éstos tengan una mejor recuperación emocional.

Así como el HJ-Biplot identifica las variables que se relacionan positiva o negativamente, también muestra aquellas variables que son independientes. En el caso de los puntajes del CEA, puede verse que las puntuaciones otorgadas a los ítems — que conforman la subescalas control emocional y regulación- fueron totalmente independientes a la puntuación proporcionada a los ítems de las subescalas recuperación y organización. Por lo tanto, intervenciones de regulación y control emocional no afectarán ni positiva ni negativamente a organización y recuperación.

Aunque no se muestran en el HJ-Biplot las variables de género, éstas resultaron ser independientes a todas las subescalas del CEA. Esto puede comprobarse por medio de las asociaciones de Spearman, donde los resultados en cada subescala van desde el -0.257 (relación entre género y organización) hasta el 0.144 (relación género con pensamiento crítico), valores que permiten asegurar que no se tienen pruebas estadísticas suficientes para indicar que las puntuaciones otorgadas a las subescalas se relacionan con el género de los estudiantes. 


\section{DISCUSIÓN Y CONCLUSIONES}

Esta investigación se enfocó en la identificación de estrategias cognitivas y estrategias metacognitivas que implementan los estudiantes de educación secundaria con aptitudes sobresalientes. Los resultados manifiestan la transferencia de la información de acuerdo con las propias experiencias personales, recordando con facilidad aquellos procedimientos aprendidos a lo largo de la formación académica. Los estudiantes con aptitudes sobresalientes recuperan información de tal forma que no requieren el uso de fuentes de búsqueda, reflejando así que tienen presentes los conocimientos para recordarlos en el momento necesario. Por tanto, la aplicación de estrategias cognitivas y metacognitivas en procedimientos más complejos les implica examinar los contenidos a detalle hasta rescatar la información deseada y determinar si es aceptable como respuesta, permitiendo reflexionar sobre la tarea a ejecutar, las posibles estrategias cognitivas para llevarla a cabo y las dificultades a las que puedan enfrentarse e, incluso, la evaluación de sus propias estrategias (Beltrán, Pérez y Ortega, 2006).

Es difícil poner punto final y concluir el trabajo de investigación descrito. No obstante, la complejidad del fenómeno de aptitudes sobresalientes saltó a la vista - una y otra vez-durante el proceso de realización de este trabajo, sobre todo durante la aplicación del «Cuestionario de estrategias de aprendizaje», porque los alumnos analizaban con detalle cada indicador y cuestionaban con frecuencia en torno de palabras poco comunes.

El trabajo pendiente es grande. Aquí hemos proporcionado algunas pautas para reflexionar pero, por delante, existen largos procesos por recorrer; desde la detección de estudiantes hasta la capacitación de los docentes, pasando por todas las variantes que esto conlleva y la constitución de múltiples y diversas estrategias con el fin de satisfacer las necesidades educativas de esta población. Con fundamento en la revisión de la literatura para la construcción del presente artículo, se identifica que la aplicación de programas de enriquecimiento cognitivo y metacognitivo, permite a la población sobresaliente aprender a aprender y ser conscientes de su capacidad de reflexión de un modo analítico, abstracto y creativo. Por 
tanto, programas educativos centrados en las estrategias cognitivas y metacognitivas los encaminan a adquirir las herramientas para el trabajo autónomo, el aprendizaje autorregulado y el control del propio aprendizaje conduciéndolos, de esta forma, a alcanzar con éxito los objetivos en cualquier área de estudio.

La reflexión generada facilita algunos elementos para seguir buscando diversas vías metodológicas de indagación, examinando aspectos relacionados con la concepción, sistematización, evaluación e intervención de los estudiantes considerados con aptitudes sobresalientes. Por tanto, los apoyos curriculares y extracurriculares deben ser claros para todos los involucrados - particularmente para los docentes-, pues son muy pocos los que participan en escuelas como la aquí descrita. La capacitación y / o actualización es aún insuficiente en educación básica (SEP, 2010).

Finalmente, como consecuencia del análisis reflexivo de los resultados, creemos importante que los docentes de educación básica guíen a los estudiantes en la adquisición e implementación de actividades que les permitan un mejor dominio en la aplicación de estrategias cognitivas y metacognitivas. Flavell (1976) afirma que:

[...] cuando pensamos en el desarrollo cognitivo, pensamos naturalmente en la adquisición de nuevas destrezas y conocimientos. Sin embargo, el desarrollo cognitivo y metacognitivo consiste también en el incremento posterior de los conocimientos y destrezas que ya existen en el repertorio (p. 166).

Al considerar esta postura, la presente investigación permite afirmar que las áreas de oportunidad de los estudiantes de educación secundaria tienen relevancia cuando no se están desarrollando; por tanto es de gran importancia intervenir implementando acciones - transversales - para que la población estudiantil desarrolle habilidades cognitivas y metacognitivas que los conduzcan a un aprendizaje autónomo. 


\section{REFERENCIAS}

Barca-Lozano, A., Almeida, L., Porto-Rioboo, A., PeralboUzquiano, M. y Brenlla-Blanco, J. (2012). Motivación escolar y rendimiento: impacto de metas académicas, de estrategias de aprendizaje y autoeficacia. Anales de Psicología, 848-859.

Beltrán, L. J. (1993). Procesos, estrategias y técnicas. Madrid: Síntesis.

Beltrán, J. y Genovard, C. (1996). Estrategias de aprendizaje. Psicología de la instrucción. Madrid: Síntesis.

Beltrán, L. J. (2003). Estrategias de aprendizaje en sujetos de altas capacidades. Educación, 55-73.

Beltrán, J., Pérez, L., Ortega, M. (2006). Manual CEA, Cuestionario de estrategias de aprendizaje. Madrid: TEA.

Davidson J. E. \& Sternberg, R.J. (1984). The role of insight in intellectual giftedness. Gifted Child Quarterly, 28, 58-64.

Diario Oficial de la Federación (30 de mayo de 2011). Ley General para la Inclusión de las Personas con Discapacidad. En http://www. sipi.siteal.iipe.unesco.org/sites/default/files/sipi normativa/ ley para la inclusion de personas con discapacidad-mexico.pdf

Flavell, J. H. (1976). Metacognitive aspects of problem solving, en Resnick, L. B. (ed) The nature of intelligence. New Jersey: Lawrence Erlbaum.

Gabriel, K. R. (1971). The Biplot Graphic Display of Matrices with Application to Principal Component Analysis. Biométrica, 58(3), 453-467. 
Galindo, M. P. y Vicente-Villardón, J. L. (1986). Una alternativa de representación simultánea: HJ-Biplot. Questiió, 10(1), 13-23.

Monereo, C. y Badia, A. (2013). Aprendizaje estratégico y tecnologías de la información y la comunicación: una revisión crítica. Revista Teoría de la educación, educación y cultura en la sociedad de la información, 14(2), 15-41.

Pifarrè, M. y Sanuy, J. (2001). La enseñanza de estrategias de resolución de problemas matemáticos en la ESO. Revista electrónica de enseñanza de las ciencias 7(19), 297-308.

Pomar, C., Díaz, O. y Fernández, M. (2006). Programa de enriquecimiento: más allá del desarrollo intelectual. La experiencia de ASAC-Galicia. Fìasca, Revista de altas capacidades, 59-66, 13.

Sánchez, I. y Pulgar, J. (2014). Impacto de un programa de renovación metodológica en las estrategias cognitivas y el rendimiento académico en cursos de física universitaria. Formación universitaria, 3-14.

Shore, B. M. (2000). Metacognitive and flexibility: qualitative differences in how gifted children think. In Friedman, R. C; Shore B. M. eds Talent unfolding: cognition and development. Washington: American Pshychological Association.

Secretaría de Educación Pública, SEP (2006). Propuesta de intervención: atención educativa a alumnos y alumnas con aptitudes sobresalientes. México: SEP.

Secretaría de Educación Pública, SEP (2010). Propuesta para la Atención Educativa de los Alumnos con Aptitudes Sobresalientes y Talentos que cursan la Educación Básica en el Estado de Guanajuato. México: SEP. 
Ugartetxea Gerrikaetxebarria, J. (1996). La orientación metacognitiva. Un estudio sobre la capacidad transferencial de la metacognición y su influencia en el rendimiento intelectual. Revista de Psicodidáctica 1, 27-53.

Valle, A., Núñez, J., Cabanach, R., Rodríguez, S., González, J. y Rosario, P. (2009). Perfiles motivacionales en estudiantes de secundaria: análisis diferencial en estrategias cognitivas, estrategias de autoregulación y rendimiento académico. Revista Mexicana de Psicología, 26(1), No. 1, 113-124.

Vicente-Villardón, J.L. (2014). MULTBIPLOT: A package for Multivariate Analysis using Biplots. Departamento de Estadística. Universidad de Salamanca. En http: / / biplot.usal.es/ClassicalBiplot/ index.html 